\title{
Analysis of the Pathogenesis of Experimental Autoimmune Optic Neuritis
}

\author{
Takeshi Kezuka, Yoshihiko Usui, and Hiroshi Goto \\ Department of Ophthalmology, Tokyo Medical University, 6-7-1 Nishi-shinjuku, Shinjuku-ku, Tokyo 160-0023, Japan \\ Correspondence should be addressed to Takeshi Kezuka, tkezuka@tokyo-med.ac.jp
}

Received 31 August 2010; Accepted 13 October 2010

Academic Editor: Oreste Gualillo

Copyright () 2011 Takeshi Kezuka et al. This is an open access article distributed under the Creative Commons Attribution License, which permits unrestricted use, distribution, and reproduction in any medium, provided the original work is properly cited.

Optic neuritis associated with multiple sclerosis has a strong association with organ-specific autoimmune disease. The goal of our research is to establish an optimal organ-specific animal model to elucidate the pathogenetic mechanisms of the disease and to develop therapeutic strategies using the model. This paper is divided into five sections: (1) clinical picture of optic neuritis associated with multiple sclerosis, (2) elucidation of pathogenesis using animal models with inflammation in optic nerve and spinal cord, (3) clinical relevance of concurrent encephalomyelitis in optic neuritis model, (4) retinal damage in a concurrent multiple sclerosis and optic neuritis model, and (5) development of novel therapies using mouse optic neuritis model. Advanced therapies using biologicals have succeeded to control intractable optic neuritis in animal models. This may ultimately lead to prevention of vision loss within a short period from acute onset of optic neuritis in human. By conducting research flexibly, ready to switch from the bench to the bedside and from the bedside to the bench as the opportunity arises, this strategy may help to guide the research of optic neuritis in the right direction.

\section{Introduction}

When ocular inflammation involves other connecting intraocular or extraocular tissues, the name of the disease changes depending on the site of inflammation. For example, the diagnosis of the disease may vary from conjunctivitis, scleritis, uveitis (intraocular inflammation), chorioretinitis, to optic neuritis. Ocular inflammation usually has an infectious or autoimmune etiology. When the cause is autoimmunity, inflammation usually involves specific organs or tissues. Our group has taken a keen interest on the study of optic neuritis associated with multiple sclerosis, which is an intractable organ-specific inflammatory disease unresponsive to conventional treatments and consequently has a poor prognosis. Optic neuritis associated with multiple sclerosis has strong association with organ-specific autoimmune disease. Our research, which aims to establish an optimal organ-specific animal model to elucidate the pathogenesis of the disease and to develop therapeutic strategies using animal model, will contribute to the understanding of the pathophysiology of intractable optic neuritis in humans and ultimately to ameliorate the disease in the future.
Optic neuritis associated with multiple sclerosis may manifest in two forms: opticospinal multiple sclerosis (OSMS) which is accompanied by cerebral lesion associated with multiple sclerosis and neuromyelitis optica (NMO) which is usually not accompanied with cerebral lesion. No conclusion has been reached on whether the two forms are in fact the same entity. The definition of NMO is the presence of optic neuritis together with spinal cord lesion extending over three or more vertebral segments, but not necessarily cerebral lesion. The presence of antibodies against aquaporin 4 (AQP4) is the most important factor associated with the pathophysiology of NMO, and it has been closed up recently $[1,2]$.

Anti-AQP4 antibody has been shown to react with AQP4 expressed on astrocytes, inducing complement fixation reaction and leading to cell death [2]. If we consider NMO as one type of autoimmune disease, B cells that are responsible for antibody production and $\mathrm{T}$ cells that regulate cytokines which promote antibody production should play important roles in the development of NMO. Although the pathogenesis of multiple sclerosis remains incompletely understood, some antigens of cerebrospinal origin, such as 
myelin basic protein (MBP), have been proposed to cause inflammation in the brain and spinal cord via some autoimmune mechanisms. At cellular level, immunity is acquired through the following pathway: antigen-antigen presenting cell-T cell-B cell. This may provide a framework to explain the clinical picture of multiple sclerosis, an autoimmune disease. Among these major cellular transmission routes, (1) the type of antigen, (2) the surface molecules (such as HLA) expressed on antigen-presenting cells including macrophages and dendritic cells, and (3) the type of lymphocytes including $\mathrm{T}$ cells and $\mathrm{B}$ cells will decide the site of inflammation and the size of the lesion. Each of these will be discussed as follows. (1) Concerning the types of protein antigen, the candidates include MBP, myelin oligodendrocyte glycoprotein (MOG), and the myelin-specific proteolipid protein (PLP). Among these proteins, MOG antigen has a high possibility of causing optic neuritis. MOG has been shown to be present abundantly inside the optic nerve, and inflammatory cells presumably react to the MOG antigen of the optic nerve to cause tissue damage [3]. In contrast, MBP is present abundantly inside the brain and spinal cord, and it has been suggested that optic neuritis rarely develops in the presence of MBP-induced encephalomyelitis. Therefore, the site of lesion is determined depending on which antigen is targeted by the immune system. (2) As for the involvement of antigen-presenting cells, classic multiple sclerosis has been associated with the expression of HLADR2 on antigen-presenting cells. In addition, the state of adhesion molecules expressed on antigen-presenting cells is a factor that attenuates inflammation. (3) Regarding the involvement of T cells and B cells, an increase in Th17-type T cells producing IFN- $\gamma$ and IL-17, as well as an accumulation of $\mathrm{B}$ cells that are precursors of the antibody-producing plasma cells have been suggested in optic neuritis associated with multiple sclerosis. In severe NMO cases in which the above-mentioned anti-AQP4 antibody was detected, apart from the autoimmune mechanisms, the antibody per se also causes direct cell damage (astroglia in this case), which may account for the higher incidence of severe damage compared to OS-MS. From the immunological point of view, it may be possible to classify anti-AQP4 antibody-positive NMO within the category of OS-MS, with the anti-AQP4 antibodynegative OS-MS classified as a mild type with little antibodyinduced tissue damage. The feasibility of this classification can only be verified in a large scale of clinical study regarding NMO.

\section{Elucidation of Pathogenetic Mechanisms Using Animal Models with Inflammations in Optic Nerve and Spinal Cord}

As discussed above, immunological mechanisms are considered to play a role in optic neuritis associated with multiple sclerosis. However, this hypothesis can only be proven when one demonstrates the development of specific lesion by adjuvant immunizing the host with the causative protein antigen. So far, research analyzing the optic nerve lesion using animal models of multiple sclerosis has not been conducted actively. In the 1990s when experimental autoimmune encephalomyelitis (EAE), which is an animal model of multiple sclerosis, was being developed, a study reported that immunizing mice with the central nervous system- (CNS-) specific PLP resulted in the onset of optic neuritis on day 17 after immunization [4]. Histopathological examination of the optic nerve showed infiltration of polymorphonuclear cells and monocytes [4], resembling the findings of optic neuritis associated with multiple sclerosis in human. Likewise, another CNS protein MOG, when used to immunize Brown Norway (BN) rats, also caused optic neuritis within 3 weeks in $90 \%$ of the animals, in addition to encephalomyelitis [5]. These studies provide clear evidence that in certain strains of rodents, optic neuritis develops as a result of immunization with antigens derived from neuron. However, even in rats and mice, the MHC types (haplotypes) in which optic neuritis can develop are probably restricted, a phenomenon probably similar to the increased susceptibility of HLA-DR2-positive human to multiple sclerosis.

In the mouse model that develops both encephalomyelitis and optic neuritis, the onset of encephalomyelitis is usually observed 13 days on average after adjuvantimmunization with the MOG35-55 peptide. However, when $\mathrm{T}$ cells obtained from these mice are injected intraperitoneally (adoptive immunization) to normal mice, optic neuritis also develops in these mice [6]. This finding indicates that the $\mathrm{T}$ cells possessing the pathogenetic factor of optic neuritis are important in the development of autoimmune optic neuritis. As an evidence that these MOG-specific T cells are an important pathogenetic factor, transgenic mice possessing $\mathrm{T}$ cells with MOG35-55-specific T cell receptor (TCR) developed optic neuritis spontaneously, with a clinical course resembling that of multiple sclerosis in humans, which is an interesting observation [7]. Furthermore, when these MOG-specific TCR transgenic mice were crossed with MOG-specific Ig heavy-chain (closely associated with B cells) knock-in mice, the incidence of spontaneous optic neuritis increased markedly [8]. As a possible mechanism, Bettelli et al. [8] proposed that IL-17- and IFN- $\gamma$-producing $\mathrm{CD}^{+} \mathrm{T}$ cells are involved in the development of optic neuritis in mice. The IL-17-secreting Th17 cells possess unique properties different from those of Th1 cells that are closely associated with the development of EAE and experimental autoimmune uveoretinitis (an animal model of human uveitis), and those of Th2 cells that are involved in allergic diseases. IL-17 has been considered as an important cytokine for the development of autoimmune diseases. Taken together, the following hypothesis may be proposed. Through the action of the newly discovered Th17 mechanism, but not Th1 or Th2, the $\mathrm{T}$ cells, IgH-producing $\mathrm{B}$ cells, and antigen that causes CNS lesion interact to produce the pathophysiology of mouse encephalomyelitis and optic neuritis.

Oligodendrocyte-specific protein- (OSP-) induced EAE is a relatively new model of multiple sclerosis, and similar findings have been reported by other institutes. OSP is a protein present in the myelin sheath of the CNS. Mice immunized with OSP develop optic neuritis together with encephalomyelitis [9]. Study has shown that OSP55-80 and 


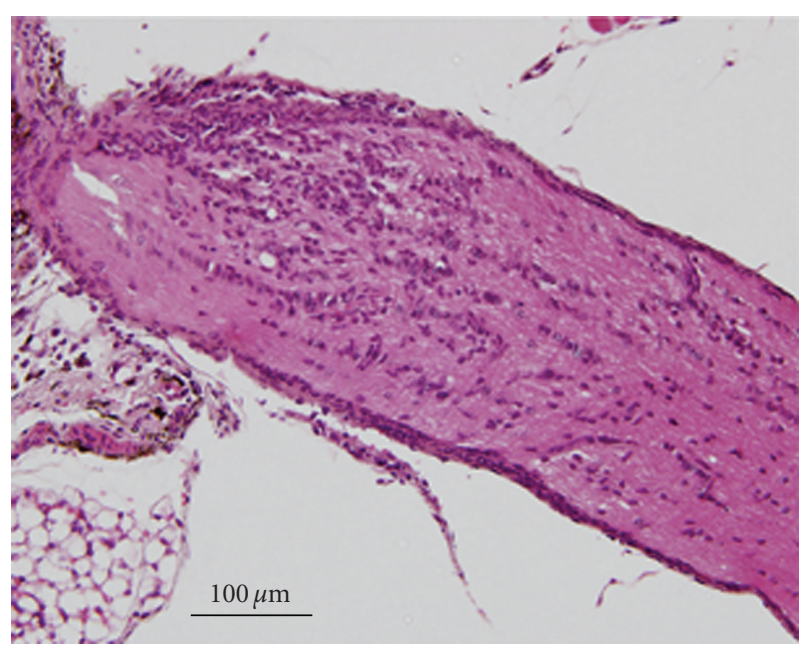

Figure 1: Histopathological findings of the optic nerve from a mouse adjuvant immunized with MOG peptide. Marked cell infiltration is observed in the optic nerve.

OSP179-207 derived from the OSP molecule are epitopes recognized by $\mathrm{CD}^{+} \mathrm{T}$ cells that mediate lesion formation. In addition, the antibodies induced by OSP were predominantly directed against OSP22-46 [9]. These findings suggest that $\mathrm{T}$ cells and $\mathrm{B}$ cells collaborate in an antigen-specific manner to give rise to the clinicopathological manifestations of this model.

From these studies, the pathogenesis of multiple sclerosis and the associated optic neuritis become gradually unraveled. However, the incidence of optic neuritis in mouse models has stayed at $60 \%$ to $70 \%$, even when gene transfer technology is used. In order to be used in therapeutic experiments in the future, a higher incidence of optic neuritis of the animal model has to be achieved. By mixing dimethyl sulfoxide (DMSO) during immunization with MOG peptide, we have successfully increased the incidence of optic neuritis from $80 \%$ to $90 \%$ in mice (report in preparation). The histopathological picture of optic neuritis obtained using this animal model is shown in Figure 1, and the Luxol myelin sheath staining of optic neuritis is shown in Figure 2. In the section stained by Luxol fast blue for myelin sheath, unstained regions indicating destruction of the myelin sheath were consistent with areas of cell infiltration. In an in vitro experiment, spleen cells collected from a mouse that developed optic neuritis were cultured, and the cytokines in the culture supernatant were assayed. IL-17 level was high, and IFN- $\gamma$ was slightly elevated in the supernatant. On the other hand, secretion of IL-10, an inhibitory cytokine, was not remarkable. These results confirm previous cytokine data of mouse optic neuritis model and probably predict the results of analysis of optic neuritis in human.

\section{Clinical Relevance of Concurrent Encephalomyelitis in Optic Neuritis Model}

When experimental autoimmune optic neuritis (EAON) is produced, EAE occurs at the same time. EAE is known to be a model of human multiple sclerosis. Regarding the relationship between EAE and multiple sclerosis, although the two share many common features histopathologically, they also differ in many aspects which remain unclarified. When we try to analyze EAON, the interpretation of EAE, which has been extensively studied, is important.

In the EAE animal model, MOG protein is considered to be the major causative antigen. In humans, approximately $50 \%$ of multiple sclerosis patients are positive for anti-MOG antibody, while an equal number of patients are negative. Recently, a tetramer radioimmunoassay has been developed, which is more sensitive than the conventional technique such as ELISA and flow cytometric assays. This radioimmunoassay is capable of detecting conformational differences in MOG protein and allows discrimination between native MOG (or folded MOG) and denatured MOG (or unfolded MOG). Human study using this method has demonstrated that antibodies from adult-onset multiple sclerosis do not bind native MOG while antibodies from children with acute disseminated encephalomyelitis (ADEM) react with native MOG. On the other hand, adult-onset multiple sclerosis cases react immunologically with denatured MOG [10]. In animal study using this method, antibodies from EAE do not bind native MOG [10].

It remains unknown whether native MOG or denatured MOG is the main target protein in autoimmune optic neuritis or NMO. If one can establish that the antibodies from optic neuritis-related diseases recognize a configuration of the MOG protein different from that recognized by antibodies from general multiple sclerosis (e.g., antibodies of patients with ADEM that recognize native MOG), this will provide evidence that optic neuritis and multiple sclerosis are independent diseases. Further analyses are expected to generate interesting new insights.

\section{Retinal Damage in a Concurrent Multiple Sclerosis and Optic Neuritis Animal Model}

In an animal model of multiple sclerosis, retinal ganglion cells are also damaged, although this finding has not been proven in humans. Meyer et al. [11] reported that encephalomyelitis developed in rats after immunization with MOG, inflammation occurred in the optic nerve axons, and apoptosis of retinal ganglion cells was induced. These results are very interesting, because it is generally believed that peripheral nerves without a myelin sheath are not attacked in multiple sclerosis. In fact, peripheral nerve fibers in the retina are not directly damaged in patients with multiple sclerosis. Likewise, a study using MOG-specific TCR transgenic mice showed that optic neuritis occurred spontaneously and retinal ganglion cells were gradually damaged starting from day 12, with TUNEL-positive apoptotic cells increasing to $83 \%$ on day 16 [12]. Therefore, in animal models of multiple sclerosis, retinal ganglion cells may be concurrently damaged, and the involvement of IL- $1 \beta$ and glial cell line-derived neurotrophic factor in neurodegeneration has attracted interest [13]. These molecules are probably related to neuroprotection. Future studies using optical coherence 


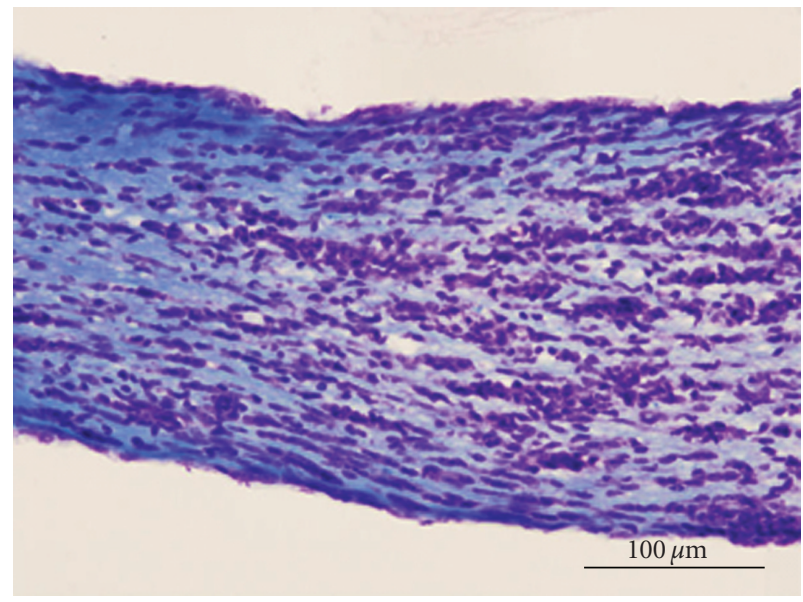

Figure 2: Luxol fast blue staining for myelin sheath in the optic nerve of a mouse adjuvant immunized with MOG peptide. Regions of weak staining of the myelin sheath corresponds to regions of cell infiltration in the optic nerve.

tomography (OCT) to analyze the perioptic nerve retina in multiple sclerosis patients may provide proof of retinal ganglion cell damage in humans.

\section{Development of Novel Therapies Using Mouse Optic Neuritis Model}

Recently, progress in the elucidation of the pathogenesis of intractable optic neuritis has reached a level that allows application of the research findings to treatment clinically. Obviously animal models are indispensable for the research and development of therapeutic modalities. Gene therapy has been attempted using animal model of optic neuritis. Guy et al. $[14,15]$ injected an adenovirus vector containing the human gene for catalase (possessing antioxidant activity) over the optic nerve head of mice with EAE and succeeded to suppress optic neuritis together with encephalomyelitis in these mice. Furthermore, by injecting an adenovirus vector containing both catalase and extracellular superoxide dismutase (ECSOD) genes (dual gene therapy) into the eye of EAE models, demyelination was attenuated $[16,17]$. Moreover, gene therapy using another antioxidant gene, superoxide dismutase 2 (SOD2), also suppressed mitochondrial oxidative stress and ameliorated the optic neuritis and encephalomyelitis in a mouse model [18].

Our group is currently conducting trials to suppress optic neuritis in mice using an approach entirely different from those reported hitherto. It is known that a specific immunoregulatory mechanism termed anterior chamber-associated immune deviation (ACAID) is present in the eye to maintain immune privilege in the intraocular environment [19]. This unique immunoregulatory mechanism in the anterior chamber maintains humoral immunity including serum antibody production, but suppresses cell-mediated immunity represented by intradermal reaction. Immunosuppressive substances such as TGF- $B$ and neuropeptides present in the anterior chamber play important roles in the ACAID phenomena. Inside the anterior chamber which is an immune privilege site, cells including macrophages are stimulated by antigen in the presence of immunoregulatory substances, and subsequently come into contact with $\mathrm{T}$ cells; these $\mathrm{T}$ cells migrate out of the eye and are transformed into regulatory $\mathrm{T}$ cells. We have examined the possibility of suppressing optic neuritis using the ACAID immunoregulatory system [20], and we found that the incidence was reduced to $70 \%$ in the group that received injection of MOG peptide antigen into the anterior chamber while $100 \%$ of mice in the positive control group developed optic neuritis. When the severity of optic neuritis was compared by histopathological scores, the anterior chamber injected group which was markedly reduced compared to the positive control group. These results prove that the ACAID immunoregulation is effective in controlling optic neuritis. The salient point about this immunoregulatory system is that disease development is mediated by the host's own immunoregulatory cells. We thus prepared immunoregulatory cells by transferring the calcitonin gene-related peptide (CGRP, known as a neuropeptide) gene into dendritic cells isolated from a mouse. Intravenous injection of the gene-transferred cells into the same mouse ameliorated optic neuritis that developed in the EAE model (report under preparation). This experimental study indicates the possibility to ameliorate optic neuritis by autologous cell therapy.

\section{Conclusion}

Research to elucidate the pathogenetic mechanism of intractable optic neuritis has shown great progress in recent years, both clinically and also in experimental models. Some of these study results have begun to reach a level permitting their application to analyses of the pathophysiology of human optic neuritis and development of treatment clinically. If cell-mediated therapy, gene therapy, and other advanced therapies using biologicals succeed to control intractable optic neuritis in animal models, this may ultimately lead to prevention of vision loss of optic neuritis in humans. By conducting research flexibly, ready to switch from the bench to the bedside and from the bedside to the bench as the opportunity arises, this strategy may help to guide the research of optic neuritis in the right direction.

\author{
Abbreviations \\ EAON: Experimental autoimmune optic neuritis \\ MS: Multiple sclerosis \\ NMO: Neuromyelitis optica \\ AQP4: Aquaporin-4 \\ MOG: Myelin oligodendrocyte glycoprotein.
}

\section{Acknowledgments}

This work was supported in part by a Grant-in-Aid for Scientific Research from the Japanese Ministry of Education and by the Human Health Science Foundation. 


\section{References}

[1] V. A. Lennon, T. J. Kryzer, S. J. Pittock, A. S. Verkman, and S. R. Hinson, "IgG marker of optic-spinal multiple sclerosis binds to the aquaporin-4 water channel," Journal of Experimental Medicine, vol. 202, no. 4, pp. 473-477, 2005.

[2] Y. Nakao, H. Yamamoto, E. Arimura et al., "Clinical features of anti-aquaporin 4 antibody-positive optic neuritis in Japanese," Neuro-Ophthalmology Japan, vol. 25, no. 3, pp. 327-342, 2008.

[3] S. F. Roemer, J. E. Parisi, V. A. Lennon et al., "Patternspecific loss of aquaporin-4 immunoreactivity distinguishes neuromyelitis optica from multiple sclerosis," Brain, vol. 130, no. 5, pp. 1194-1205, 2007.

[4] N. T. Potter and P. E. Bigazzi, "Acute optic neuritis associated with immunization with the CNS myelin proteolipid protein," Investigative Ophthalmology and Visual Science, vol. 33, no. 5, pp. 1717-1722, 1992.

[5] M. K. Storch, A. Stefferl, U. Brehm et al., "Autoimmunity to myelin oligodendrocyte glycoprotein in rats mimics the spectrum of multiple sclerosis pathology," Brain Pathology, vol. 8, no. 4, pp. 681-694, 1998.

[6] H. Shao, Z. Huang, S. L. Sun, H. J. Kaplan, and D. Sun, "Myelin/oligodendrocyte glycoprotein-specific T-cells induce severe optic neuritis in the C57B1/6 mouse," Investigative Ophthalmology and Visual Science, vol. 45, no. 11, pp. 4060 4065, 2004.

[7] E. Bettelli, M. Pagany, H. L. Weiner, C. Linington, R. A. Sobel, and V. K. Kuchroo, "Myelin oligodendrocyte glycoproteinspecific $\mathrm{T}$ cell receptor transgenic mice develop spontaneous autoimmune optic neuritis," Journal of Experimental Medicine, vol. 197, no. 9, pp. 1073-1081, 2003.

[8] E. Bettelli, D. Baeten, A. Jäger, R. A. Sobel, and V. K. Kuchroo, "Myelin oligodendrocyte glycoprotein-specific T and B cells cooperate to induce a Devic-like disease in mice," Journal of Clinical Investigation, vol. 116, no. 9, pp. 2393-2402, 2006.

[9] N. Kaushansky, M.-C. Zhong, N. K. De Rosbo, R. Hoeftberger, H. Lassmann, and A. Ben-Nun, "Epitope specificity of autoreactive $\mathrm{T}$ and $\mathrm{B}$ cells associated with experimental autoimmune encephalomyelitis and optic neuritis induced by oligodendrocyte-specific protein in SJL/J mice," Journal of Immunology, vol. 177, no. 10, pp. 7364-7376, 2006.

[10] K. C. O’Connor, K. A. McLaughlin, P. L. De Jager et al., "Selfantigen tetramers discriminate between myelin autoantibodies to native or denatured protein," Nature Medicine, vol. 13, no. 2, pp. 211-217, 2007.

[11] R. Meyer, R. Weissert, R. Diem et al., "Acute neuronal apoptosis in a rat model of multiple sclerosis," Journal of Neuroscience, vol. 21, no. 16, pp. 6214-6220, 2001.

[12] Y. Guan, K. S. Shindler, P. Tabuena, and A. M. Rostami, "Retinal ganglion cell damage induced by spontaneous autoimmune optic neuritis in MOG-specific TCR transgenic mice," Journal of Neuroimmunology, vol. 178, no. 1-2, pp. 4048, 2006.

[13] M. B. Sättler, M. Togni, I. Gadjanski et al., "Strain-specific susceptibility for neurodegeneration in a rat model of autoimmune optic neuritis," Journal of Neuroimmunology, vol. 193, no. 1-2, pp. 77-86, 2008.

[14] J. Guy, X. Qi, and W. W. Hauswirth, "Adeno-associated viral-mediated catalase expression suppresses optic neuritis in experimental allergic encephalomyelitis," Proceedings of the National Academy of Sciences of the United States of America, vol. 95, no. 23, pp. 13847-13852, 1998.

[15] J. Guy, X. Qi, H. Wang, and W. W. Hauswirth, "Adenoviral gene therapy with catalase suppresses experimental optic neuritis," Archives of Ophthalmology, vol. 117, no. 11, pp. 1533-1539, 1999.

[16] X. Qi, W. W. Hauswirth, and J. Guy, "Dual gene therapy with extracellular superoxide dismutase and catalase attenuates experimental optic neuritis," Molecular Vision, vol. 13, pp. 111, 2007.

[17] X. Qi, L. Sun, A. S. Lewin, W. W. Hauswirth, and J. Guy, "Long-term suppression of neurodegeneration in chronic experimental optic neuritis: antioxidant gene therapy," Investigative Ophthalmology and Visual Science, vol. 48, no. 12, pp. 5360-5370, 2007.

[18] X. Qi, A. S. Lewin, L. Sun, W. W. Hauswirth, and J. Guy, "Suppression of mitochondrial oxidative stress provides long-term neuroprotection in experimental optic neuritis," Investigative Ophthalmology and Visual Science, vol. 48, no. 2, pp. 681-691, 2007.

[19] J. W. Streilein, "Ocular immune privilege: therapeutic opportunities from an experiment of nature," Nature Reviews Immunology, vol. 3, no. 11, pp. 879-889, 2003.

[20] Y. Matsunaga, T. Kezuka, and Y. Usui, "Immune privilege ameliorates murine experimental autoimmune optic neuritis induced with myelin/oligodendrocyte glycoprotein," in Proceedings of the Annual Meeting of Association for Research in Vision and Ophthalmology (ARVO '08), April 2008. 


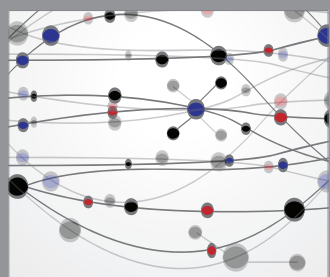

The Scientific World Journal
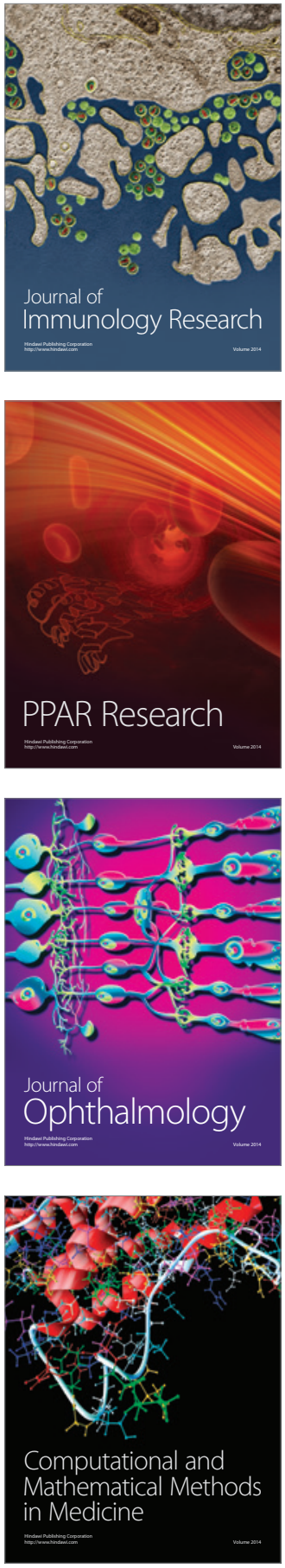

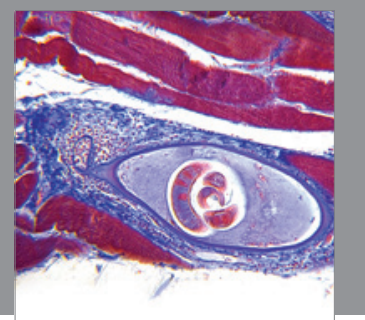

Gastroenterology

Research and Practice
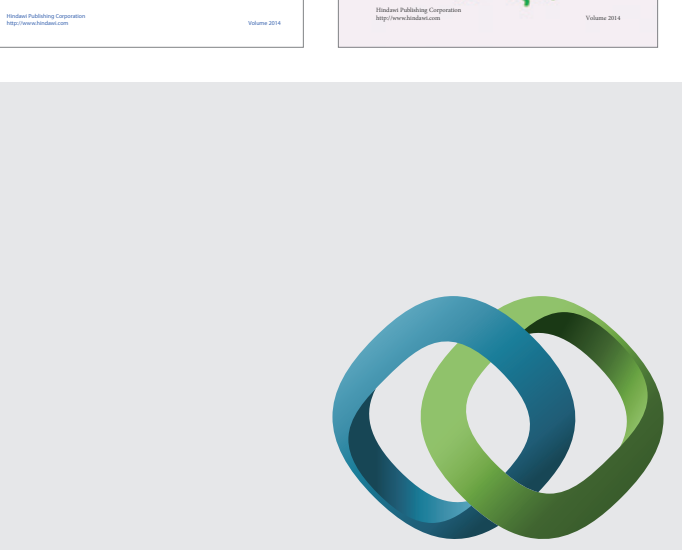

\section{Hindawi}

Submit your manuscripts at

http://www.hindawi.com
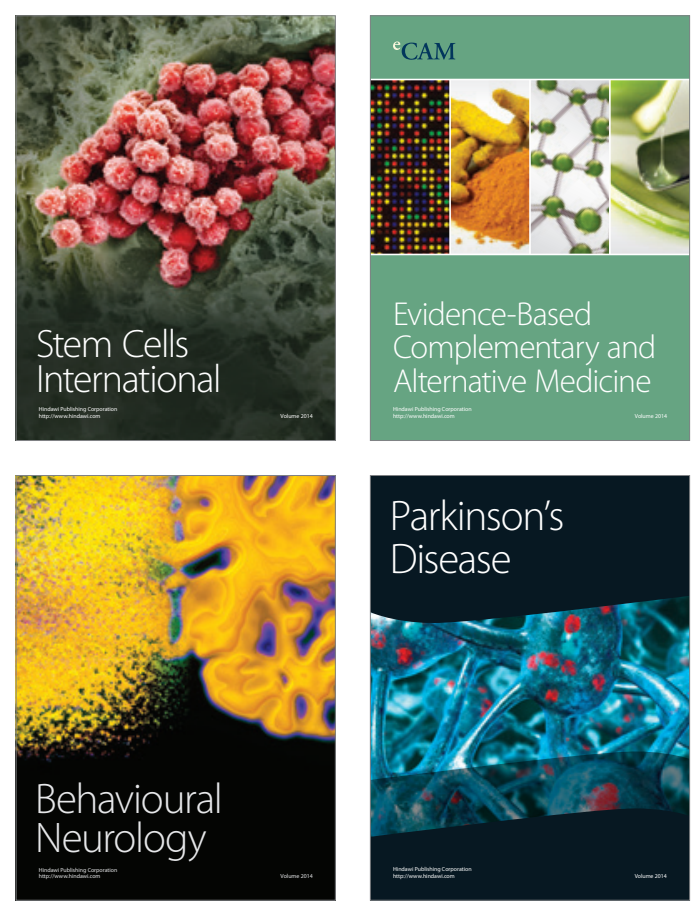

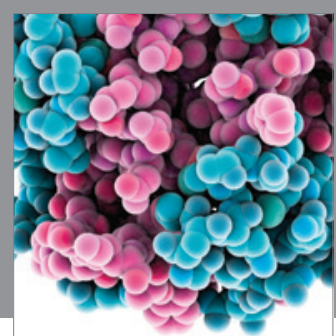

Journal of
Diabetes Research

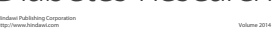

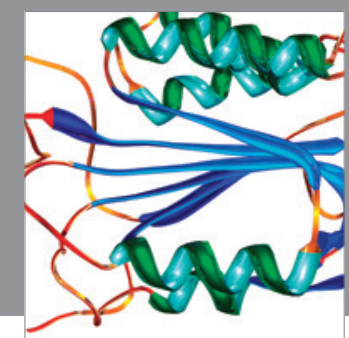

Disease Markers
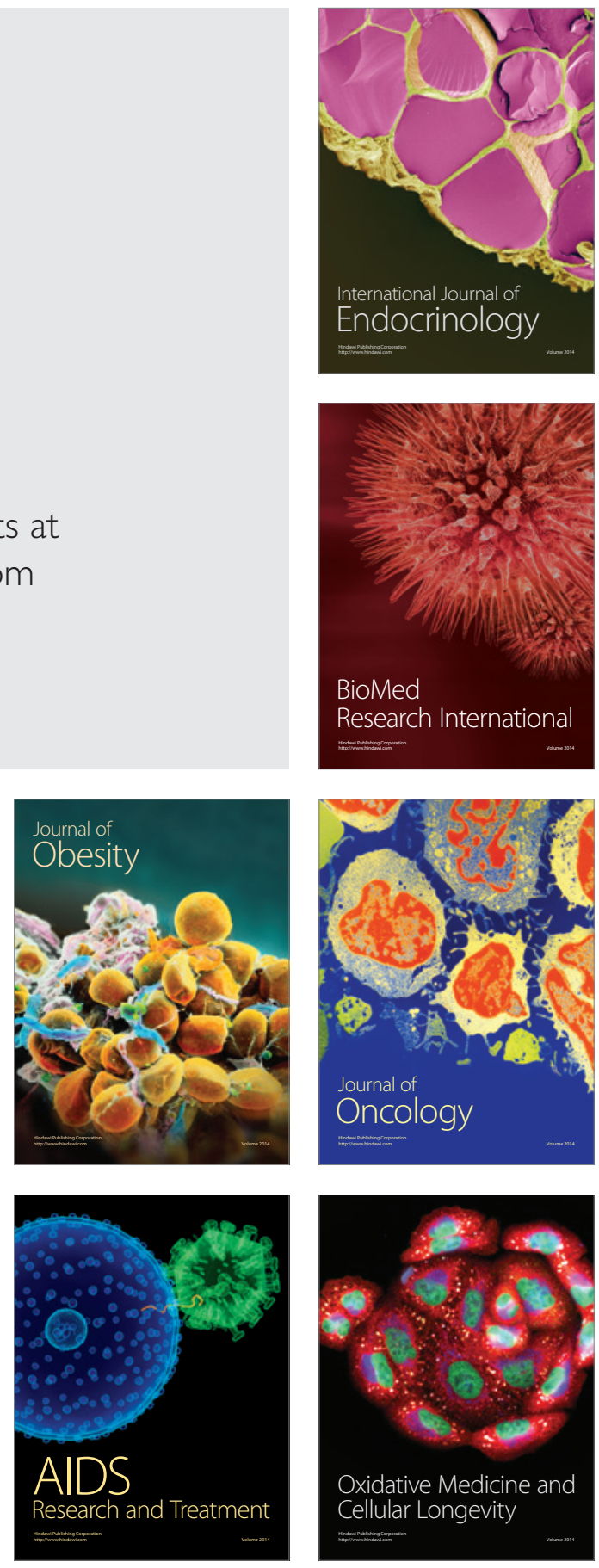